\title{
Escherichia coli RuvA and RuvB proteins specifically interact with Holliday junctions and promote branch migration
}

\author{
Hiroshi Iwasaki, Masahiko Takahagi, Atsuo Nakata, and Hideo Shinagawa ${ }^{1}$ \\ Department of Experimental Chemotherapy, Research Institute for Microbial Diseases, Osaka University, \\ Suita, Osaka 565, Japan
}

\begin{abstract}
The Escherichia coli ruvA and ruvB genes are involved in DNA repair and in the late step of homologous genetic recombination. We have demonstrated previously that the RuvA-RuvB protein complex in the presence of ATP promotes reabsorption of cruciform structures extruded from a supercoiled plasmid with an inverted repeat sequence. Because the cruciform structure is topologically analogous to the Holliday structure, we have proposed that the role of the RuvA and RuvB proteins in recombination is to promote a strand exchange reaction at the Holliday junction. Here, we studied the specific interaction of the RuvA-RuvB complex with the Holliday structure using synthetic analogs prepared by annealing four oligonucleotides. The affinities of the RuvA protein for synthetic Holliday junctions are much higher ( $>20$-fold) than for duplex DNA, and the affinities of the RuvA protein for the junctions are further enhanced ( $>4$-fold) by the interaction with the RuvB protein. The RuvA-RuvB protein complex in the presence of ATP promotes dissociation of the synthetic Holliday junction with homology in the central core into two halves by catalyzing branch migration to the DNA ends, but it does not affect the structure of the synthetic Holliday junction without the homology. The separation of the synthetic Holliday junction is a result of the activity of the RuvA-RuvB complex that promotes strand exchange and DNA unwinding. Furthermore, RuvA and RuvB promote the strand exchange reaction at the Holliday junctions made by RecA. These results provide further evidence that the RuvA-RuvB complex recognizes the Holliday junction and promotes branch migration in homologous recombination.
\end{abstract}

[Key Words: Branch migration; helicase; Holliday junction; recombination; RecA protein; RuvA-RuvB protein complex]

Received June 26, 1992; revised version accepted August 27, 1992.

Escherichia coli cells with a mutation in any of the three $\operatorname{ruv}$ genes, $\operatorname{ruv} A, \operatorname{ruv} B$, and $\operatorname{ruv} C$, are sensitive to various DNA-damaging agents such as UV irradiation, $\mathrm{X}$-rays, and chemical mutagens (Otsuji et al. 1974; Lloyd et al. 1984; Iwasaki et al. 1989a; Sargentini and Smith 1989; Sharples et al. 1990). Although the ruv single mutants are nearly as proficient as the wild-type strain in genetic recombination, the ruv derivatives of $r e c B C s b c A, r e c B C$ $s b c B C$, and recG are severely defective in recombination and highly sensitive to DNA-damaging agents (Lloyd 1991; Lloyd et al. 1984, 1987). These findings suggest that the products of $\operatorname{ruv} A, \operatorname{ruv} B$, and $\operatorname{ruv} C$ are involved in recombination repair of damaged DNA. In addition, the $\operatorname{ruv} A$ and $\operatorname{ruv} B$ mutants have some defects in X-ray- and UV-induced mutagenesis (Sargentini and Smith 1989; $\mathrm{H}$. Iwasaki, T. Kato, and H. Shinagawa, unpubl.). The ruvA

${ }^{1}$ Corresponding author. and $\operatorname{ruvB}$ genes constitute a LexA-regulated operon (Shurvinton and Lloyd 1982; Benson et al. 1988; Shinagawa et al. 1988), whereas the ruvC gene located near the ruvA-ruvB operon is not regulated by the SOS system (Sharples et al. 1990; Sharples and Lloyd 1991; Takahagi et al. 1991). Like the recA gene, the ruvA and $r u v B$ genes apparently play multiple roles in recombination, repair, and mutagenesis.

The RuvC protein has been purified recently and shown to be an endonuclease that resolves the Holliday structure (Dunderdale et al. 1991; Iwasaki et al. 1991), which is a proposed intermediate of homologous recombination (Holliday 1964). The RuvA and RuvB proteins have been overproduced and purified to near homogeneity, and their properties have been studied (Iwasaki et al. 1989b; Shiba et al. 1991). The RuvB protein possesses weak ATPase activity, which is greatly enhanced by the RuvA protein bound to DNA. In the presence of ATP, the RuvA-RuvB protein complex promotes reabsorption 
of a cruciform structure that is formed by extrusion of an inverted repeat sequence in supercoiled DNA. Because the cruciform structure is structurally analogous to the Holliday structure in which two homologous duplex molecules are linked by a single-stranded crossover, and the reabsorption of the cruciform involves exchanges of single-stranded partners between the homologous pairs of duplex DNA, we proposed previously that the role of the RuvA and RuvB proteins in homologous recombination is to interact specifically with the Holliday junction and promote branch migration (Iwasaki et al. 1991; Shiba et al. 1991; Shinagawa et al. 1991).

In this work we studied specific interactions of the RuvA protein and the RuvA-RuvB protein complex with synthetic Holliday junctions and with the Holliday structures made by RecA and obtained further evidence that supports the above hypothesis.

\section{Results}

RuvA protein binds preferentially to the synthetic Holliday junction

Because the RuvA-RuvB protein complex promotes, with accompanying ATP hydrolysis, reabsorption of the cruciform extruded from supercoiled DNA with an inverted repeat, and the RuvA protein itself is a DNAbinding protein (Shiba et al. 1991), we wanted to show that RuvA actually binds to the Holliday junction. We prepared synthetic four-way junctions by annealing four nucleotide oligomers as analogs of the Holliday junction (Fig. 1) and examined by gel-retardation assay whether the RuvA protein binds preferentially to the Holliday junction with a central homology core of $12 \mathrm{bp}$ (Fig. 2A). RuvA formed complexes with the Holliday junction (Fig. $2 \mathrm{~A}$, lanes 11-15) in the concentration range that allowed no complex formation with the linear duplex DNA (lane
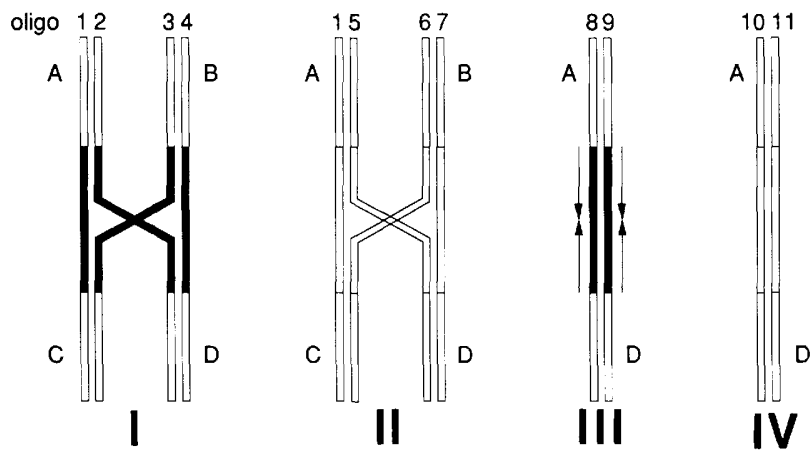

Figure 1. Schematic drawing of synthetic Holliday junctions and duplex DNAs used in this study. One of the synthetic Holliday junctions (I) contains a homology core of $12 \mathrm{bp}$ (solid bars) flanked by heterologous sequences A, B, C, and D, which are shared by the Holliday junction that does not contain homologous sequences (II). The duplex DNA with inverted repeat sequences (III) contains a 12-bp inverted repeat (solid bars) flanked by $\mathrm{A}$ and $\mathrm{D}$ sequences, which are shared with the duplex DNA without inverted repeat sequences (IV).
1-5). The affinity of the RuvA protein for the synthetic Holliday junction was 20-fold or more than that for the duplex DNA, as estimated by the amount of RuvA needed to give equivalent binding.

\section{RuvB protein enhances the affinity of RuvA protein for the Holliday junction}

As the DNA-bound RuvA protein enhances the ATPase activity of the RuvB protein (Shiba et al. 1991), we wanted to determine whether RuvB enhances DNAbinding activity of RuvA. As shown in Figure 2A, RuvA in the presence of excess RuvB exhibited approximately fourfold higher affinity for the junction DNA than it did in the absence of RuvB. Because a RuvA tetramer forms a stable complex with a RuvB dimer in solution /Shinagawa et al. 1991), and the relative electrophoretic mobilities of the protein-DNA complexes formed in the presence and in the absence of the RuvB protein were the same, the RuvB protein appears to be dissociated from the RuvA-DNA complex during electrophoresis under the present experimental conditions. RuvB also appears to enhance the affinity of RuvA for the duplex DNA (Fig. 2A, lanes 6-10).

Effect of the homology core in the junction on the interaction with the RuvA-RuvB protein complex

The effect of the homology core in the junction on the interaction with the RuvA-RuvB complex was studied by comparing relative affinities of the synthetic Holliday junctions with and without a homology core for the protein complex. The RuvA-RuvB complex showed similar affinity for the junction DNA with or without the homology core in the repeated experiments. It bound to both of the junction DNAs with much higher affinity ( $\sim 20$-fold) than to the duplex DNAs with or without the inverted repeat sequence (Fig. 2B). Therefore, the RuvARuvB protein complex interacts preferentially with Holliday junctions, and the strength of this interaction appears to be largely dependent on the topology of DNA.

The RuvA-RuvB protein complex dissociates the Holliday junction by promoting branch migration

We examined the effect of nucleotides on the interaction between the RuvA-RuvB protein complex and the synthetic Holliday junction by gel retardation assay. No effect of nucleotides ATP, ADP, or ATP $(\gamma S)$ on the binding affinity of the RuvA-RuvB protein complex for the junction was observed (data not shown). However, a new DNA band, which migrated faster than the junction DNA, was observed by incubation with ATP but not with ADP or ATP $(\gamma S)$. We then analyzed by gel electrophoresis the DNA products after the reaction products were treated with EDTA and SDS to dissociate protein from DNA (Fig. 3A). The position of the DNA band (lane 7) between the junction and the duplex DNA bands corresponded to that of a half-cruciform (marker 3 in lane 3). Therefore, we concluded that incubation of the junction 
Figure 2. Gel-retardation assay of RuvA binding to Holliday junctions. $|A|$ Specific binding of the RuvA protein to the synthetic Holliday junction and its enhancement by the RuvB protein. DNAbinding activity of the RuvA protein in the absence (lanes $1-5,11-15$ ) or in the presence (lanes $6-10$, $16-20)$ of the RuvB protein ( $860 \mathrm{nM}$ ) was examined by gel-retardation assay. In lanes $1-10,{ }^{32} \mathrm{P}$-labeled duplex DNA with an inverted repeat sequence 167 $\mathrm{nM})$, and in lanes $11-20,{ }^{32} \mathrm{P}$-labeled synthetic Holliday junction with homology cores $(67 \mathrm{nM})$ were used as target DNAs. Lanes 1, 6, 11, and 16 contained no RuvA; lanes 2, 7, 12, and 17 contained 27 nM of RuvA; lanes $3,8,13$, and 18 contained $68 \mathrm{nM}$ of RuvA; lanes $4,9,14$, and 19 contained $340 \mathrm{~nm}$ of RuvA; lanes 5, 10,15, and 20 contained $680 \mathrm{~nm}$ of RuvA. $(B)$ Effect of homology cores in the junction on the interaction with the RuvA-RuvB complex. Binding affinity of the RuvA protein for the duplexes (lanes 1-10) and the synthetic junctions (lanes 11-20) was examined by gel-retardation assay. The RuvB protein ( $340 \mathrm{nM}$ ) was included in all reaction mixtures. Lanes $1,6,11$, and 16 contained no RuvA; lanes 2, 7, 12, and 17 contained $6.8 \mathrm{nM}$ of RuvA; lanes $3,8,13$, and 18 contained $13.6 \mathrm{nM}$ of RuvA; lanes 4, 9, 14, and 19 contained $34 \mathrm{~nm}$ of RuvA; lanes $5,10,15$, and 20 contained $136 \mathrm{~nm}$ of RuvA.

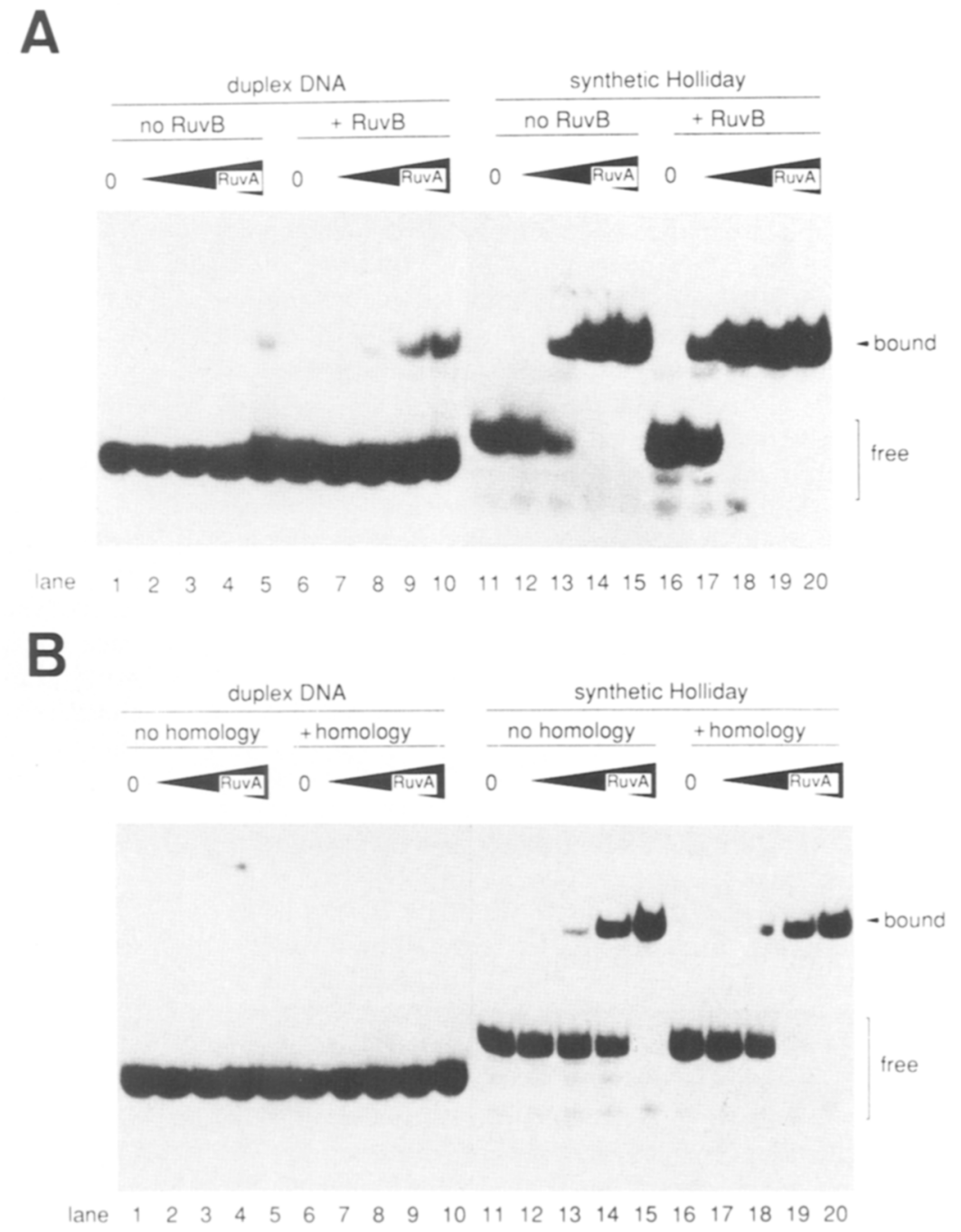

DNA with RuvA and RuvB in the presence of ATP resulted in the dissociation of the junction. The reaction requires both the RuvA and RuvB proteins and ATP hydrolysis (Fig. 3A). We then examined the substrate specificity of this reaction (Fig. 3B). The RuvA-RuvB complex catalyzed dissociation of the synthetic Holliday junction with the homology core but did not alter the structure of the junction without the homology or that of the duplex DNAs. This reaction is shown schematically in Figure 4. The RuvA-RuvB complex binds specifically to the crossover junctions, promotes a strand exchange reaction in the central core of homology, and then unwinds the remainder of the DNA to the ends by helicase activity, using the energy of ATP hydrolysis.

The RuvA-RuvB protein complex dissociates the Holliday junctions made by RecA by promoting branch migration

Because the RuvA-RuvB-mediated strand exchange reaction with the synthetic Holliday junctions involves only a very short region of homology (12 bp), we wanted to determine whether RuvA and RuvB could promote strand exchange reaction between much longer homologous duplex DNAs. For this purpose, we employed the Holliday junctions made by RecA between gapped circular DNA and homologous linear duplex DNA (Müller et al. 1990) as the substrate for the RuvA-RuvB-mediated strand exchange reaction (Fig. 5). We examined whether RuvA and RuvB could promote dissociation of the Holliday junctions that were made by RecA and subsequently deproteinized. The formation of Holliday junctions was confirmed by cleavage with the RuvC protein (Fig. 6, lane 1), which is a Holliday junction-specific endonuclease (Dunderdale et al. 1991; Iwasaki et al. 1991). RuvA and RuvB dissociated the Holliday junctions by promoting branch migration, and this reaction required both the RuvA and RuvB proteins and ATP (Fig. 6), as in the case of the dissociation of the synthetic Holliday junctions (Fig. 3). When we added RuvA and RuvB to the linear and gapped DNAs in the presence of ATP, we could not detect the formation of the recombination intermediates (Holliday junctions) or the products of the strand exchange reaction (data not shown), indicating 

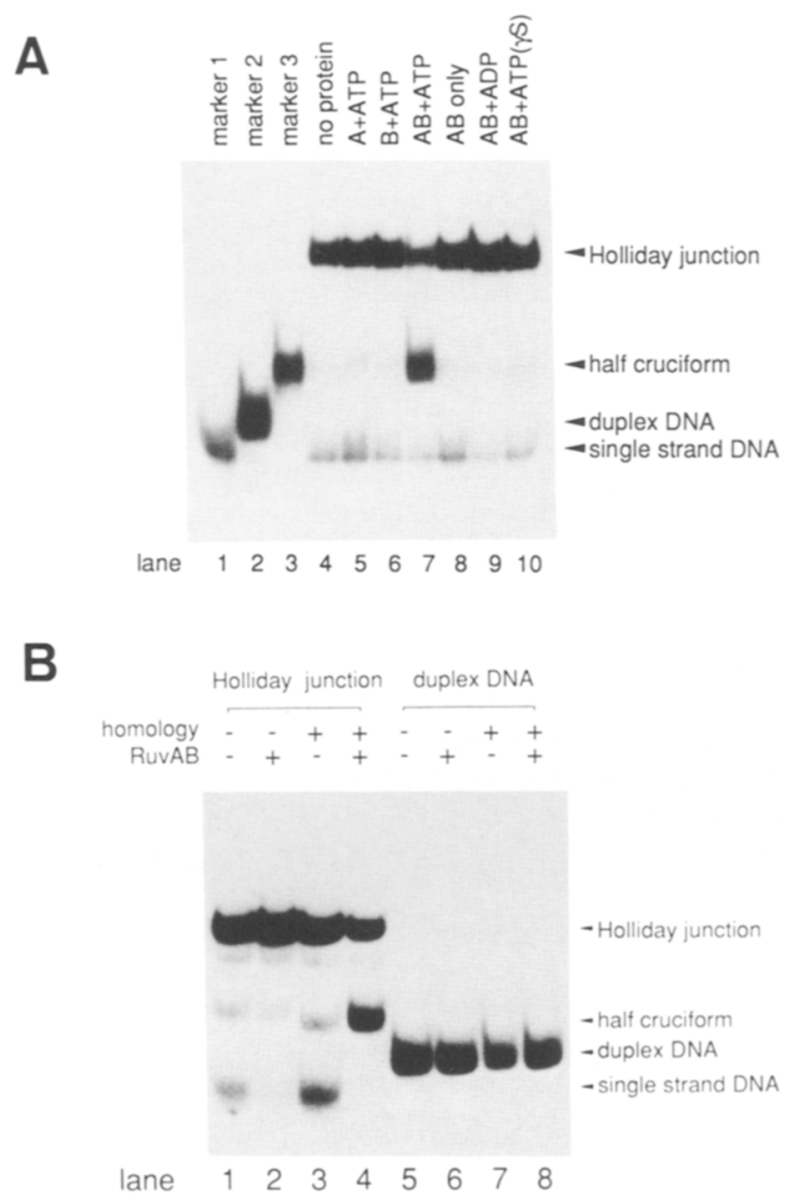

Figure 3. Dissociation of the synthetic Holliday junction by the RuvA-RuvB protein complex. $(A)$ Dissociation of the Holliday junction requires both the RuvA and RuvB proteins and ATP. The synthetic Holliday junction with homology (67 $\mathrm{nM}$ ) was incubated with RuvA (68 nM) and RuvB (43 nM) as indicated in the presence $(2 \mathrm{mM})$ or in the absence of nucleotide at $37^{\circ} \mathrm{C}$ for $10 \mathrm{~min}$. The reaction products were analyzed after deproteinization with SDS and EDTA by electrophoresis in a $9 \%$ polyacrylamide gel. (Lane 1) Single-stranded DNA (oligonucleotide 1) as a marker; (lane 2) a marker for duplex DNA, which was prepared by annealing oligonucleotides 8 and 9; (lane 3) a marker containing a half cruciform, which was prepared by annealing oligonucleotides 1 and 2; (lane 4) no protein; (lane 5) RuvA + ATP; (lane 6) RuvB + ATP; (lane 7) RuvA and RuvB + ATP; (lane 8) RuvA and RuvB without nucleotide; (lane 9) RuvA and RuvB + ADP; (lane 10) RuvA and RuvB + ATP $(\gamma S)$. (B) The RuvA-RuvB protein complex dissociates the Holliday junction with homology. Various forms of DNA were incubated with the RuvA and RuvB proteins (lanes 2, 4, 6, 8) or without the proteins (lanes $1,3,5,7$ ), and the reaction products were analyzed as above. (Lanes 1,2$)$ The synthetic Holliday junction without homology; (lanes 3,4) the synthetic Holliday junction with homology; (lanes 5,6) duplex DNA without an inverted repeat; (lanes 7,8 ) duplex DNA with an inverted repeat.

that RuvA and RuvB cannot initiate the strand exchange reaction as RecA does.

\section{Discussion}

The RuvA and RuvB proteins in the presence of ATP promote reabsorption of a cruciform structure extruded from a supercoiled plasmid with an inverted repeat sequence. The ATPase activity of RuvB is enhanced by RuvA bound to DNA. The UV irradiation of supercoiled DNA further enhances this stimulatory effect of the supercoiled DNA on ATPase activity, but UV irradiation has no effect on the stimulation by the linear duplex DNA. To explain these phenomena, we proposed that UV adducts induce cruciform structures in the supercoiled DNA, with which RuvA interacts to enhance the ATPase activity of RuvB (Shiba et al. 1991). From these observations, we also proposed that the RuvA-RuvB complex specifically interacts with the Holliday junction and promotes branch migration (Iwasaki et al. 1991; Shiba et al. 1991; Shinagawa et al. 1991). Consistent with this hypothesis, RuvA binds to synthetic Holliday junctions with much higher affinity than to linear duplex DNA. Although the two junction DNAs with or without homology core appear to possess equivalent topology, only the former has branch migration potential, which is closer in property to the true Holliday structure.

The RuvA and RuvB proteins exert allosteric effects on each other; RuvA bound to DNA enhances the ATPase activity of RuvB as shown previously by Shiba et al. (1991), and RuvB enhances the DNA-binding activity of RuvA (Fig. 2A). The addition of ATP, ADP, or ATP $(\gamma S)$ had no effect on the affinity of the RuvA-RuvB complex for the synthetic Holliday junctions. However, the RuvA-RuvB protein complex in the presence of ATP promoted dissociation of the synthetic Holliday junction (Fig. 3A). This reaction can be arbitrarily separated into two steps: The first step involves a strand exchange reaction between the homologous duplex in the core, and the second step involves unwinding of the duplex without concomitant rewinding in the rest of the region, which requires helicase activity. The RuvA-RuvB protein complex in the presence of ATP did not dissociate the duplex DNAs nor the synthetic Holliday junction without the central core of homology (Fig. 3B). These results support the hypothesis that the role of RuvA and RuvB proteins in homologous recombination is to interact specifically with the Holliday junctions and to promote branch migration.

The RecA protein in the presence of ATP promotes the formation of the Holliday junction between homologous gapped circular DNA (gDNA) and linear duplex DNA and promotes branch migration at the junction (Müller et al. 1990). The RuvA and RuvB proteins in the presence of ATP also promote branch migration of the Holliday junction that is formed by the RecA protein (Fig. 6). Therefore, although the RecA protein promotes both pairing and strand exchange reactions between homologous DNAs (for a review, see Radding 1988), the RuvA and RuvB proteins also likely play a role in branch migration. Based on the failure to recover transconjugants of F-prime factor in ruv derivatives of $\operatorname{rec} B C \quad s b c B C$ strains, Benson et al. (1991) proposed that the RuvABC proteins function in a late step of recombination in which they process recombination intermediates into vi- 
Figure 4. Schematic drawing of the dissociation of the synthetic Holliday junction by the RuvA-RuvB protein complex with accompanying ATP hydrolysis. The RuvA-RuvB complex in the presence of ATP promotes the strand exchange reaction in the region of the homologous core and then unwinding of the rest of the nonhomologous region.

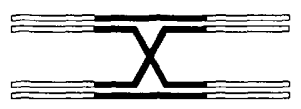

synthetic Holliday junction (cruciform)

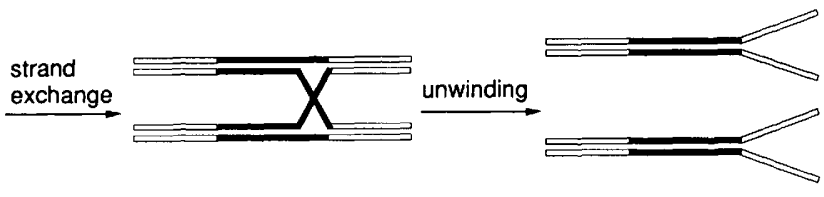

(half-cruciform) able products. We interpret this observation in the light of the present work as follows. Newly entering F-prime factor in the recipient cell is highly recombinogenic, because it requires synthesis of the lagging strand and, consequently, contains gapped or single-stranded regions. The RecA protein promotes pairing and initiates strand exchanges between F-prime DNA and chromosomal DNA, resulting in the formation of the DNA complex with single-stranded crossovers (Holliday structure). The helicase activity of the RuvA-RuvB complex is required for dissociation of the entangled DNA formed by RecA; and, finally, the crossover junctions are resolved by the Holliday junction-specific endonuclease, RuvC protein. Although both RecA and RuvA-RuvB appear to promote branch migration, they may play different roles in recombination. The RecA does not bind to synthetic Holliday junctions while RuvA-RuvB does not initiate strand exchanges between gDNA and linear duplex DNA.

Although ruv or recG mutants are only slightly defective in homologous recombination, $\operatorname{ruv} A \operatorname{rec} G$, ruv $B$ rec $G$, and ruvC rec $G$ double mutants are very defective in recombination, indicating functional overlap between the RecG and Ruv proteins (Lloyd 1991). The RecG protein, deduced from the sequence of the recG gene, possesses sequences homologous to the consensus domains of several DNA and RNA helicases (Lloyd and Sharples 1991). These findings suggest that the RecG protein also interacts with Holliday junctions and promotes branch migration as the RuvA-RuvB complex does.

ruv mutant cells are longer than isogenic $\mathrm{ruv}^{+}$cells, and they elongate to form multinucleate filamentous cells when exposed to low doses of DNA-damaging agents. The ability of ruv mutants to form filament cells is dependent on the sfiA gene function, which negatively regulates the septum formation (Otsuji et al. 1974; Otsuji and Iyehara-Ogawa 1979; Lloyd et al. 1984). One interpretation of these results is that the SOS-inducing signal accumulates more in ruv mutants, and the filamentation is a manifestation of the overexpression of the SOS-inducible $s$ fiA gene. Treatments that damage DNA produce recombinogenic structures in DNA, and RecA initiates recombination but fails to complete the process in the absence of any functions of $\operatorname{ruvABC}$. This leads to accumulation of recombination intermediates in the cell. These recombination intermediates formed by RecA, which are accumulated more in the absence of the ruv functions, may be equivalent to the RecA-DNA complexes (activated RecA) that promote cleavage of the LexA repressor.

Recently, it has been demonstrated independently that the RuvA-RuvB complex specifically interacts with Holliday junctions (Parsons et al. 1992) and promotes branch migration (Tsaneva et al. 1992). Taylor (1992) has recently reviewed the roles of the $\operatorname{ruv} A, \operatorname{ruv} B, \operatorname{ruv} C$, and $r e c G$ genes in migration and resolution of Holliday junctions.

\section{Materials and methods}

RuvA, RuvB, and RuvC proteins

The RuvA (Shiba et al. 1991; $>97 \%$ pure), RuvB (Iwasaki et al. $1989 \mathrm{~b}$; $>98 \%$ pure), and RuvC proteins (Iwasaki et al. 1991; $>99 \%$ pure) were used. Protein concentration was determined by the Bradford method with a Bio-Rad protein assay kit.

\section{Other proteins}

Restriction endonucleases and DNA modification enzymes were obtained from Takara Shuzo (Kyoto). The RecA protein was generously given by Dr. Horii (Research Institute for Microbial Diseases).

\section{Preparation of synthetic Holliday junction analogs}

Oligonucleotides were synthesized by the phosphoramidite
Figure 5. Schematic drawing of the formation of the Holliday junctions by RecA and the dissociation of the junctions by RuvA and RuvB. The substrates are gDNA and ${ }^{32} \mathrm{P}$-end-labeled linear duplex DNA. The RecA protein promotes homologous pairing and strand exchange reactions to form the recombination intermediates (Holliday structure) and products $\left({ }^{32} \mathrm{P}-\mathrm{la}\right.$ beled nicked circular and ${ }^{32}$ p-labeled gapped linear DNA).

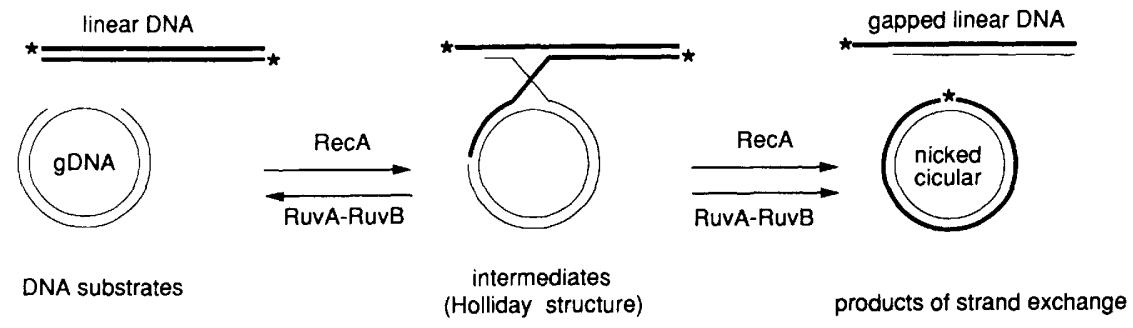




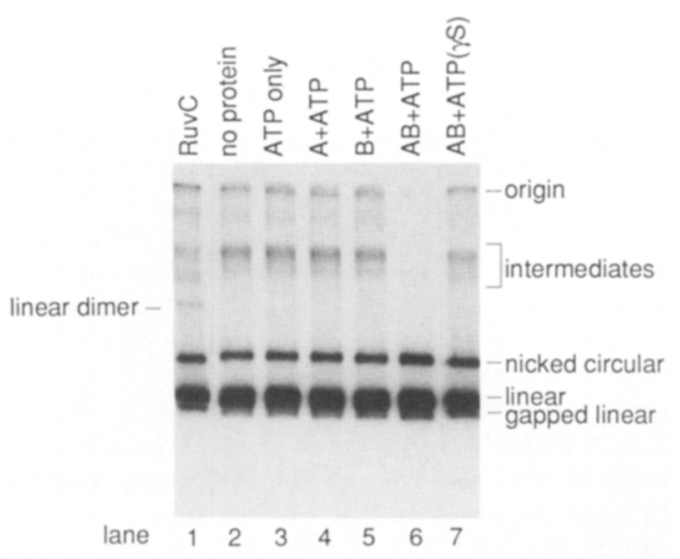

Figure 6. Dissociation of the Holliday junctions by the RuvARuvB-mediated branch migration. Recombination intermediates between gDNA and homologous ${ }^{32} \mathrm{P}$-labeled linear duplex DNA were formed by RecA and used as substrates for the RuvA-RuvB-mediated branch migration after deproteinization, as described in Materials and methods. The reaction products were analyzed by agarose gel electrophoresis after deproteinization and visualized by autoradiography. In lane 1 , the reaction mixture contained $0.1 \mu \mathrm{M}$ of RuvC.

method with an Applied Biosystems 380B DNA synthesizer and purified by high-performance liquid chromatography (HPLC) with reverse-phase column chromotography. Synthetic Holliday junctions were prepared by annealing four strands of 49-mer oligonucleotides as described by Parsons et al. (1990). Oligonucleotides were labeled at the $5^{\prime}$ terminus using $\left[\gamma^{-32} \mathrm{P}\right] \mathrm{ATP}$ and T4 polynucleotide kinase. The synthetic Holliday junction, which contains a central core of homology of $12 \mathrm{bp}$ flanked by heterologous sequence, was prepared by annealing oligonucleotides 1 (5'-ATCGATGTCTCTAGACAGCTGCTCAGGATTGATCTGTAATGGCCTGGGA-3'), 2 (5'-GTCCCAGGCCATTACAGATCAATCCTGAGCATGTTTACCAAGCGCATTG$\left.3^{\prime}\right), 3$ (5'-TGATCACTTGCTAGCGTCGCAATCCTGAGCAGCTGTCTAGAGACATCGA-3'), and 4 (5'-CCAATGCGCTTGGTAAACATGCTCAGGATTGCGACGCTAGCAAGTGATC-3'). The synthetic Holliday junction without a central core of homology was prepared by annealing oligonucleotides 1 , 5 (5'-GTCCCAGGCCATTACAGATCAATCCACTATGTGTTTACCAAGCGCATTG-3'), 6 (5'-TGATCACTTGCTAGCGTCGATTCGGTGAGCAGCTGTCTAGAGACATCGA-3' $\mid$, and 7 (5'-CC-AATGCGCTTGGTAAACACATAGTCCGAATCGACGCTAGCAAGTGATC-3'). Linear duplex DNA with a 2-bp inverted repeat sequence in the center was prepared by annealing oligonucleotides 8 (5' ${ }^{\prime}$-TGATCACTTGCTAGCGTCGCAATCCGGATTGATCTGTAATGGCCTGGGA-3' $)$ and 9 (5'GTCCCAGGCCATTACAGATCAATCCGGATTGCGACGCTAGCAAGTGATC-3'). Linear duplex DNA without an inverted repeat sequence was prepared by annealing oligonucleotides 10 (5'-TGATCACTTGCTAGCGTCGATTCGGGGATTGATCTGTAATGGCCTGGGA-3') and 11 (5'-GTCCCAGGCCATTACAGATCAATCCCCGAATCGACGCTAGCAAGTGATC-3'). The DNA concentrations were measured at 260 $\mathrm{nm}$ and expressed in terms of molar concentrations of junction or duplex DNA.

\section{Protein-DNA-binding assay}

Varying amounts of the RuvA protein or the RuvA protein plus the RuvB protein were incubated with $5^{\prime}-{ }_{-}^{32} \mathrm{P}$-labeled synthetic
Holliday junction or duplex DNA (67 $\mathrm{nM}$ of DNA) for $10 \mathrm{~min}$ at $37^{\circ} \mathrm{C}$ in $10 \mu$ l of buffer $\mathrm{H}$ containing $20 \mathrm{~mm}$ HEPES-KOH $(\mathrm{pH}$ 7.6), $10 \mathrm{~mm} \mathrm{Mg-acetate,} 7 \mathrm{~mm} \beta$-mercaptoethanol, and $5 \%$ glycerol. To each sample, $2 \mu \mathrm{l}$ of sample solution $(15 \%$ glycerol and $0.1 \%$ bromphenol blue) was added and the complex formation was assayed by electrophoresis in a $5 \%$ polyacrylamide gel. Electrophoresis was carried out at $4^{\circ} \mathrm{C}$ at $100 \mathrm{~V}$ for $1 \mathrm{hr}$ in a buffer containing Tris-acetate $(\mathrm{pH} 8.0)$ and $1 \mathrm{~mm}$ EDTA. Gels were dried, and DNA bands were visualized by autoradiography.

\section{Dissociation of synthetic Holliday junctions}

A standard reaction mixture $(20 \mu l)$ containing the RuvA protein ( $68 \mathrm{nM}$ ), the RuvB protein (43 nM), substrate DNA (67 nM), and ATP $(2 \mathrm{mM})$ in buffer $\mathrm{H}$ was incubated at $37^{\circ} \mathrm{C}$ for $10 \mathrm{~min}$. Reactions were stopped by adding $4 \mu \mathrm{l}$ of stop buffer containing $20 \mathrm{~mm}$ Tris- $\mathrm{HCl}$ (pH 7.5), $100 \mathrm{~mm}$ EDTA, $1 \%$ SDS, $0.25 \%$ bromphenol blue, $0.25 \%$ xylene cyanol, and $30 \%$ glycerol. The reaction products were analyzed by $9 \%$ polyacrylamide gel electrophoresis at room temperature and visualized by autoradiography.

\section{Preparation of recombination intermediates}

gDNA was prepared by annealing single-stranded DNA of phasimid pUC119 and $2.8 \mathrm{~kb}$ of NaeI-HincII double-stranded pUC119 essentially as described by Müller et al. (1990) who used $\phi X 174$ DNA instead of pUC119. The strand exchange reaction $(100 \mu \mathrm{l})$ was performed as described (Müller et al. 1990) using gDNA (12 $\mu \mathrm{M}$ as nucleotide concentration) and $5^{\prime}{ }^{32} \mathrm{P}$ labeled linear duplex DNA (10 $\mu \mathrm{M})$, which had been cut with HincII. After incubation for $12 \mathrm{~min}$ at $37^{\circ} \mathrm{C}$, the reaction was stopped by adding SDS $(0.5 \%)$, EDTA $(50 \mathrm{~mm})$, and proteinase $\mathrm{K}$ $(2 \mathrm{mg} / \mathrm{ml})$ and incubated for an additional $30 \mathrm{~min}$ at $37^{\circ} \mathrm{C}$. The mixture was deproteinized twice with phenol-chloroform, and the DNA was precipitated with ethanol. After being dried, the DNA was suspended in $40 \mu \mathrm{l}$ of a buffer containing $10 \mathrm{~mm}$ HEPES- $\mathrm{KOH}$ and $1 \mathrm{mM}$ EDTA.

\section{Promotion of branch migration at the Holliday junctions by RuvA and RuvB}

A reaction mixture (10 $\mu$ l) containing RuvA (68 nM), RuvB (43 $\mathrm{nM})$, substrate DNA $(2 \mu \mathrm{l})$ prepared as described above, and ATP $(2 \mathrm{mM})$ or $\mathrm{ATP}(\gamma \mathrm{S})(2 \mathrm{mM})$ in buffer $\mathrm{H}$ was incubated at $37^{\circ} \mathrm{C}$ for $10 \mathrm{~min}$. Reactions were stopped by adding SDS $(0.5 \%)$, EDTA (50 mM), and proteinase $\mathrm{K}(2 \mathrm{mg} / \mathrm{ml})$ and incubated for an additional $10 \mathrm{~min}$. The products were analyzed by $1.4 \%$ agarose gel electrophoresis, and visualized by autoradiography.

\section{Acknowledgments}

We thank B. Benton for the critical reading of our manuscript. This work was supported by a Grants-in-Aid for Scientific Research from the Ministry of Science, Education, and Culture of Japan to H.S. and H.I., and by Research Aid of the Inoue Foundation for Science to H.I.

The publication costs of this article were defrayed in part by payment of page charges. This article must therefore be hereby marked "advertisement" in accordance with 18 USC section 1734 solely to indicate this fact.

\section{References}

Benson, F.E., G.T. Illing, G.J. Sharples, and R.G. Lloyd. 1988. Nucleotide sequencing of the ruv region of Escherichia coli 
$\mathrm{K}-12$ reveals a LexA regulated operon encoding two genes. Nucleic Acids Res. 16: 1541-1549.

Benson, F., S. Collier, and R.G. Lloyd. 1991. Evidence of abortive recombination in ruv mutants of Escherichia coli K12. Mol. Gen. \& Genet. 225: 266-272.

Dunderdale, H.J., F.E. Benson, C.A. Parsons, G.J. Sharples, R.G. Lloyd, and S.C. West. 1991. Formation and resolution of recombination intermediates by $E$. coli RecA and RuvC proteins. Nature 354: 506-510.

Holliday, R. 1964. A mechanism for gene conversion in fungi. Genet. Res. 5: 282-304.

Iwasaki, H., T. Shiba, A. Nakata, and H. Shinagawa. 1989a. Involvement in DNA repair of the ruvA gene of Escherichia coli. Mol. \& Gen. Genet. 219: 328-331.

- 1989b. Overproduction, purification, and ATPase activity of the Escherichia coli RuvB protein involved in DNA repair. I. Bacteriol. 171: 5276-5280.

Iwasaki, H., M. Takahagi, T. Shiba, A. Nakata, and H. Shinagawa. 1991. Escherichia coli RuvC protein is an endonuclease that resolves the Holliday structure. EMBO $\mathrm{J}$. 10: $4381-4389$.

Lloyd, R.G. 1991. Conjugational recombination in resolvasedeficient ruvC mutants of Escherichia coli K-12 depends on recG. J. Bacteriol. 173: 5414-5418.

Lloyd, R.G. and G.J. Sharples. 1991. Molecular organization and nucleotide sequence of the recG locus of Escherichia coli K-12. J. Bacteriol. 173: 6837-6843.

Lloyd, R.G., F.E. Benson, and C.E. Shurvinton. 1984. Effect of ruv mutations on recombination and DNA repair in Escherichia coli Kl2. Mol. \& Gen. Genet., 194: 303-309.

Lloyd, R.G., C. Buckman, and F.E. Benson. 1987. Genetic analysis of conjugational recombination in Escherichia coli $\mathrm{Kl} 2$ strains deficient in RecBCD enzyme. J. Gen. Microbiol. 133: 2531-2538.

Müller, B., C. Jones, B. Kemper, and S.C. West. 1990. Enzymatic formation and resolution of Holliday junctions in vitro. Cell 60: 329-336.

Otsuii, N., and H. Iyehara-Ogawa. 1979. Thermoresistant revertants of an Escherichia coli strain carrying tif-1 and ruv mutations: Non-suppressibility of ruv by sfi. I. Bacteriol. 138: $1-6$.

Otsuij, N., H. Iyehara, and Y. Hideshima. 1974. Isolation and characterization of an Escherichia coli ruv mutant which forms nonseptate filaments after low doses of ultraviolet light irradiation. I. Bacteriol. 117: 337-344.

Parsons, C.A., B. Kemper, and S.C. West. 1990. Interaction of a four-way junction in DNA with T4 endonuclease VII. J. Biol. Chem. 265: 9285-9289.

Parsons C.A., I. Tsaneva, R.G. Lloyd, and S.C. West. 1992. Interaction of Escherichia coli RuvA and RuvB proteins with synthetic Holliday junctions. Proc. Natl. Acad. Sci. 89: 5452-5456.

Radding, C.M. 1988. Homologous pairing and strand exchange promoted by Escherichia coli RecA protein. In Genetic recombination (ed. R.Kucherlapati and G.R. Smith), pp. 193229. American Society for Microbiology, Washington, D.C.

Sargentini, N.J. and K.C. Smith. 1989. Role of ruvAB genes in $\mathrm{UV}$ - and $\gamma$-radiation and chemical mutagenesis in Escherichia coli. Mutat. Res. 215: 115-129.

Sharples, G.J. and R.G. Lloyd. 1991. Resolution of Holliday junctions in Escherichia coli: Identification of the ruvC gene product as a 19-kilodalton protein. I. Bacteriol. 173: 77117715.

Sharples, G.J., F.E. Benson, G.T. Illing, and R.G. Lloyd. 1990. Molecular and functional analysis of the ruv region of Escherichia coli $\mathrm{K}-12$ reveals three genes involved in DNA repair and recombination. Mol.\& Gen. Genet. 221: 219-226.

Shiba, T., H. Iwasaki, A. Nakata, and H. Shinagawa. 1991. SOSinducible DNA repair proteins, RuvA and RuvB, of Escherichia coli: Functional interactions between RuvA and RuvB for ATP hydrolysis and renaturation of the cruciform structure in supercoiled DNA. Proc. Nat1. Acad. Sci.. 88: 84458449.

Shinagawa, H., K. Makino, M. Amemura, S. Kimura, H. Iwasaki, and A. Nakata. 1988. Structure and regulation of the Escherichia coli ruv operon involved in DNA repair and recombination. I. Bacteriol. 170: 4322-4329.

Shinagawa, H., T. Shiba, H. Iwasaki, K. Makino, M. Takahagi, and A. Nakata. 1991. Properties of the Escherichia coli RuvA and RuvB proteins involved in DNA repair, recombination and mutagenesis. Biochimie 73: 505-507.

Shurvinton, C.E. and R.G. Lloyd. 1982. Damage to DNA induces expression of the ruv gene of Escherichia coli. Mol. \& Gen. Genet. 185: 352-355.

Takahagi, M., H. Iwasaki, A. Nakata, and H. Shinagawa. 1991. Molecular analysis of the Escherichia coli ruvC gene, which encodes a Holliday junction-specific endonuclease. $J$. Bacteriol. 173: 5747-5753.

Taylor, A.F. 1992. Movement and resolution of Holliday junctions by enzymes from E. coli. Cell 69: 1063-1065.

Tsaneva, I.R., B. Mller, and S.C. West. 1992. ATP-dependent branch migration of Holliday junctions promoted by the RuvA and RuvB proteins of E. coli. Cell 69: 1171-1180. 


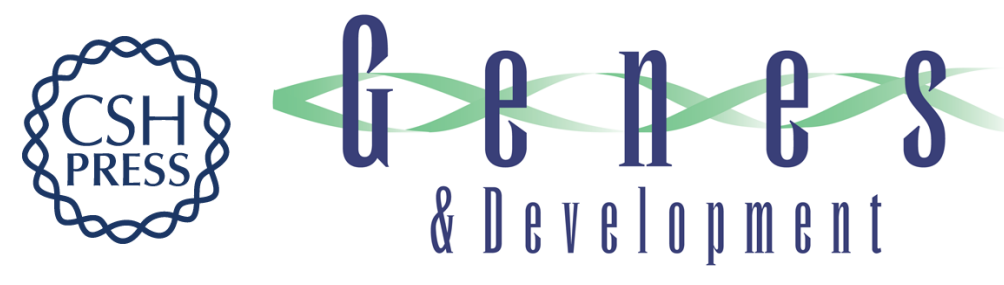

\section{Escherichia coli RuvA and RuvB proteins specifically interact with Holliday junctions and promote branch migration.}

H Iwasaki, M Takahagi, A Nakata, et al.

Genes Dev. 1992, 6:

Access the most recent version at doi:10.1101/gad.6.11.2214

References This article cites 26 articles, 11 of which can be accessed free at: http://genesdev.cshlp.org/content/6/11/2214.full.html\#ref-list-1

License

Email Alerting

Receive free email alerts when new articles cite this article - sign up in the box at the top Service right corner of the article or click here.

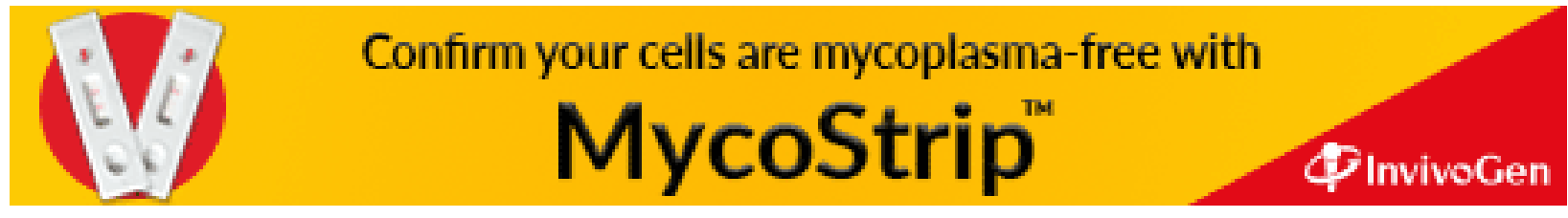

